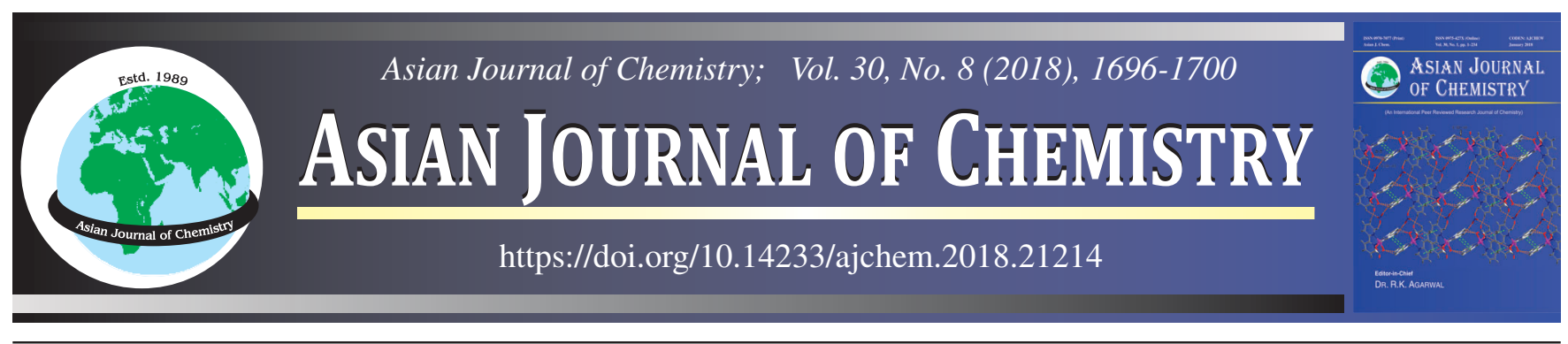

\title{
Synthesis and Characterization of Pure and Triethanolamine Capped Hydroxyapatite Nanoparticles and its Antimicrobial and Cytotoxic Activities
}

V. Kalaiselvi ${ }^{1,2, *}$ and R. Mathammal ${ }^{1}$

${ }^{1}$ Department of Physics, Sri Sarada College for Women, Salem-636 016, India

${ }^{2}$ Department of Physics, Navarasam Arts \& Science College for Women, Erode-638 101, India

*Corresponding author: E-mail: nk.arthikalai@gmail.com

Received: 6 January 2018; Accepted: 6 June 2018;

Published online: 30 June 2018;

AJC-18958

Pure hydroxyapatite and triethanolamine capped hydroxyapatite nanoparticles were synthesized by microwave irradiation method. The nanoparticle size, morphology, phase purity and elemental composition were analyzed by XRD, SEM, TEM, FTIR and EDAX. The samples were further analyzed by antimicrobial and cytotoxity test. The XRD peaks are narrow indicated that the crystalline phase of pure hydroxyapatite with $42 \mathrm{~nm}$ and triethanolamine capped hydroxyapatite nanoparticles with $22 \mathrm{~nm}$ and is in good agreement with JCPDS card with number 09-0432. The morphology was identified to be spherical shaped by SEM micrographs with diameter of around 40-32 $\mathrm{nm}$. The TEM images show the formation of smallest particles with diameter less than $50 \mathrm{~nm}$. FTIR results showed the characteristic peak of $\mathrm{O}=\mathrm{H}$ and $\mathrm{P}=\mathrm{O}$ bond. EDAX demonstrates that it has standard $\mathrm{Ca} / \mathrm{P}$ ratio 1.6. Antibacterial behaviour of pure and triethanolamine capped hydroxyapatite were investigated by agar well diffusion method using gram negative bacilli Escherichia coli and gram positive cocci Staphylococcus aureus which showed a considerable activity. The cytotoxicity was performed by using human embryonic kidney cell line HEK 293 which revealed the non-cytotoxic nature of triethanolamine capped hydroxyapatite nanoparticles towards HEK293 cell line.

Keywords: Biomaterials, Triethanolamine, Hydroxyapatite, Capping, Microwave irradiation.

\section{INTRODUCTION}

Hydroxyapatite $\left.\left[\mathrm{Ca}_{10}\left(\mathrm{PO}_{4}\right)_{6}(\mathrm{OH})_{2}\right)\right]$ is a bioactive biomaterial, equivalent to mineral phase in bone and widely used due to its biocompatible property [1]. Nano-hydroxyapatite is particularly applied in DNA and drug delivery for cells. To maintain structure, control of size, composition should be concentrated during synthesis of nanoscale materials. Porous structured hydroxyapatite improves the drug loading capacity, calcium deficient product enhances restorability in cells and bodies are the basic identity of the material [2].

Hydroxyapatite plays a vital role in dentistry as tooth enamel and orthopedics as bone mineral. It is biocompatible, bioactive, non-toxic, osteoconductive and non-inflammatory [3]. It belongs to apatite family and widely applied in repair, fill, expand and reconstruct the damaged tissue. It forms good chemical bond with hard host tissue, hence used in medical field as implants, as coating during prostheses, bone filling material. Narrow structured biomaterials are extensively applied in all rooms of medicine. Hydroxyapatite with morphologically controlled structures is preferred in the medical field as biosensors, photocatalysts, drug delivery and pollution control agents [4].
Many chemical methods have been employed in synthesizing fine hydroxyapatite powders namely sol-gel method, microwave method, chemical precipitation method, controlled precipitation, etc. [5]. Microwave irradiation method is the useful method to control the size, morphology, structure and homogeneity of hydroxyapatite nanoparticles due to the restriction during gel growth. The calcium deficient composites can be prepared by this method at room temperature. This is uncomplicated method, less energy and time consuming and produces highly pure form of nano hydroxyapatite [6]. In the present work, an unique version of nanosized hydroxyapatite were synthesized by adopting less expensive host materials which helps for bulk preparation of hydroxyapatite. An attempt has been made to prepare pure and triethanolamine capped hydroxyapatite nanoparticles by microwave irradiation $[1,7]$. The effect of triethanolamine capping on hydroxyapatite plays a vital role in structural, morphological and composition of hydroxyapatite nanoparticles were also investigated [8]. Among various capping agents, triethanolamine is applied to produce hydroxyapatite with reduced particle size, toxicity and avoids agglomeration of the synthesized sample. This report clearly deals with biological applications by capping hydroxyapatite nanoparticles via microwave irradiation [9]. 


\section{EXPERIMENTAL}

All the analytical grade chemicals used in this study were procured from Sigma-Aldrich Company. The XRD was performed in Bruker D8 Advance Diffractometer. It has one dimensional detector performed in integrated mode. It is nickel filtered with $\mathrm{CuK} \alpha$ radiation (Shimadzu, Model-XRD 6000, Japan). Fourier transform infrared spectroscopy analysis was carried out to identify the functional groups and the spectrum was recorded in 4000-400 $\mathrm{cm}^{-1}$ region by $\mathrm{KBr}$ method. The shape and surface morphology and elemental composition were identified using scanning electron microscopy and EDAX (JEOL, ModelJSM 6390, Japan).The particle size and morphology were further examined using TEM [10].

Synthesis of hydroxyapatite nanopowders: Pure hydroxyapatite nanopowders were synthesized by microwave irradiation with calcium hydroxide and orthophosphoric acid as host precursors. Disperse appropriate amount of calcium hydroxide in $100 \mathrm{~mL}$ distilled water to get one mole of $\mathrm{Ca}(\mathrm{OH})_{2}$ solution. Similarly, $0.6 \mathrm{M}$ orthophosphoric acid solution was diluted by adding appropriate amount of orthophosphoric acid in $100 \mathrm{~mL}$ double distilled water. Since hydroxyapatite has $\mathrm{Ca}: \mathrm{P}$ ratio as $1: 67,0.6 \mathrm{M}$ orthophosphoric acid solution was incorporated dropwise to the calcium hydroxide solution. To obtain homogeneity the dropping rate must be well controlled. The solution was stirred for 30 mins and obtained a gelatinous white precipitate. The sodium hydroxide solution was added in drops while stirring to obtain $\mathrm{pH} 8$. The final precipitate gets stirred for next $4 \mathrm{~h}$. The precipitate was aged for $24 \mathrm{~h}$. The aged sol was washed with double distilled water and finally with ethanol to remove the impurities from residual solution. The final precipitate was placed in domestic microwave oven for $10 \mathrm{~min}$ at $70 \mathrm{~W}$. when the water molecules get precipitated, increased the temperature to $500 \mathrm{~W}$. The dried cake was placed in the furnace at $250{ }^{\circ} \mathrm{C}$ and made into very fine powder using mortar. The capped sample was prepared by dispersing calcium hydroxide solution in $0.1 \mathrm{M}$ triethanolamine solution instead of distilled water.

Antimicrobial activity: The antibacterial activity was performed with the two standard clinical strains such as $S$. aureus (Gram positive) and E. coli (Gram negative) using a agar well diffusion method. The nutrient agar plate was used for the inoculation of both bacterial strains. The bacterial strains were swabbed using cotton buds or swab and about $7 \mathrm{~mm}$ diameter of well was made using a well borer. About $25 \mu \mathrm{L}$ of synthesized samples were added into the well and plates placed in an incubator at room temperature for $24 \mathrm{~h}$. Standard ciprofloxacin was used as positive control while water as negative control to perform the study [11,12].

Cytotoxicity test: The MTT assay test was performed to test cytotoxicity of different nanomaterials. The human embryonic kidney cell line was received from National center of cell science (NCCS) and grown in fetal bovine serum (FBS) in eagle minimum essential medium. To make single suspensions the monolayer was detached by using trypsin-EDTA. A mitochondrial enzyme in living cells cleaves by making purple formazon into insoluble product. The obtained product and viable cells are directly proportional to each other.The MTT and DMSO are used for further purification using micro-plate reader after incubation of $48 \mathrm{~h}[13,14]$. The percentage of cell growth and control was calculated using the following formula:

$$
\text { Cell growth }(\%)=\frac{\text { Test }[\mathrm{A}]}{\operatorname{Control}[\mathrm{A}]} \times 100
$$

\section{RESULTS AND DISCUSSION}

FT-IR analysis: FTIR spectrum (Fig. 1) clearly shows the presence of various vibrational phosphates and hydroxyl groups. The peaks at 1037, 1095 and $1029 \mathrm{~cm}^{-1}$ corresponds to the asymmetric stretching mode of $\mathrm{P}-\mathrm{O}$ bond in the apatite $\mathrm{PO}_{4}{ }^{3-}$ group. The wavenumber peaks 604,569 and $462 \mathrm{~cm}^{-1}$ represent the bending modes of O-P-O bond. The shift in hydroxyl peaks at $3413 \mathrm{~cm}^{-1}$ in pure hydroxyapatite and 3437 $\mathrm{cm}^{-1}$ in triethanolamine substituted hydroxyapatite occurs due to the shift in hydrogen bond in triethanolamine which is blue shifted to standard FTIR. The appearance of sharp peak at $873 \mathrm{~cm}^{-1}$ was associated with the carbonate group and indicates the presence of carbonates in the sample. The presence of $\mathrm{CO}_{3}{ }^{2-}$ is a consequence of atmospheric $\mathrm{CO}_{2}$ interacting with hydroxyapatite precursors in the solution during synthesis. The presence of triethanolamine in dried hydroxyapatite samples are revealed by $-\mathrm{CH}_{2}$ group, present at two weak peaks at 2928 and 2854 $\mathrm{cm}^{-1}$. The interaction between hydroxyapatite and triethanolamine disappears due to the combination of this shift and -OH stretching mode enhancement at $3437 \mathrm{~cm}^{-1}$.

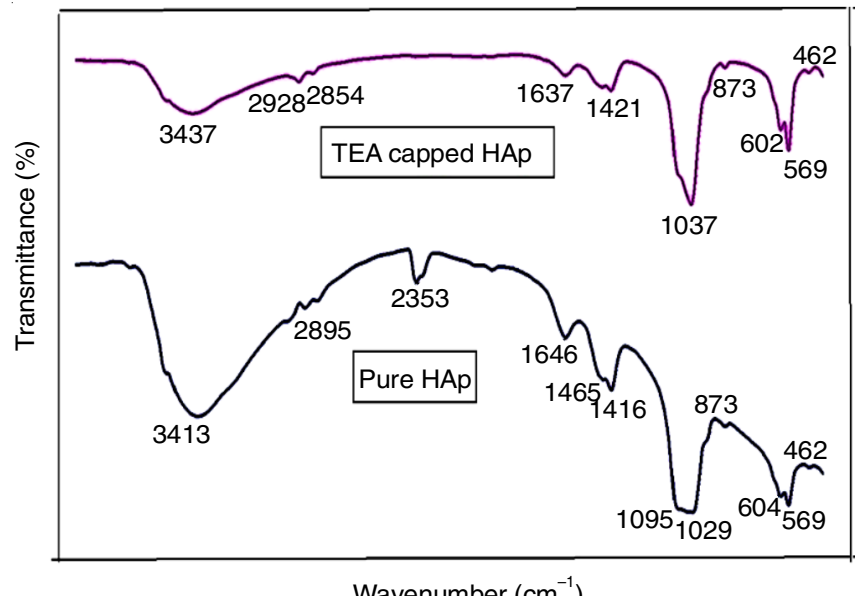

Fig. 1. FTIR spectra of pure and triethanolamine capped hydroxyapatite nanopowders

XRD analysis: Fig. 2 shows the XRD pattern of hydroxyapatite nanoparticles with and without triethanolamine capping. Both pure and capped samples exhibits the hexagonal structure, $\mathrm{P}_{3} / m$ symmetry, well matched with JCPDS card no. 09-0432. In triethanolamine capping sample, broadening of peaks and decrease in peak intensities were observed. The average crystalline sizes of hydroxyapatite nanoparticles was determined by using Scherrer's equation:

$$
\mathrm{d}=\frac{\mathrm{K} \lambda}{\beta \cos \theta}
$$

where $\mathrm{d}$ is the mean crystallite size, $\mathrm{K}=0.89$ is the shape factor, $\lambda$ is the wavelength of the incident beam, $\beta$ is the full width at half maximum and $\theta$ is the Bragg angle. 


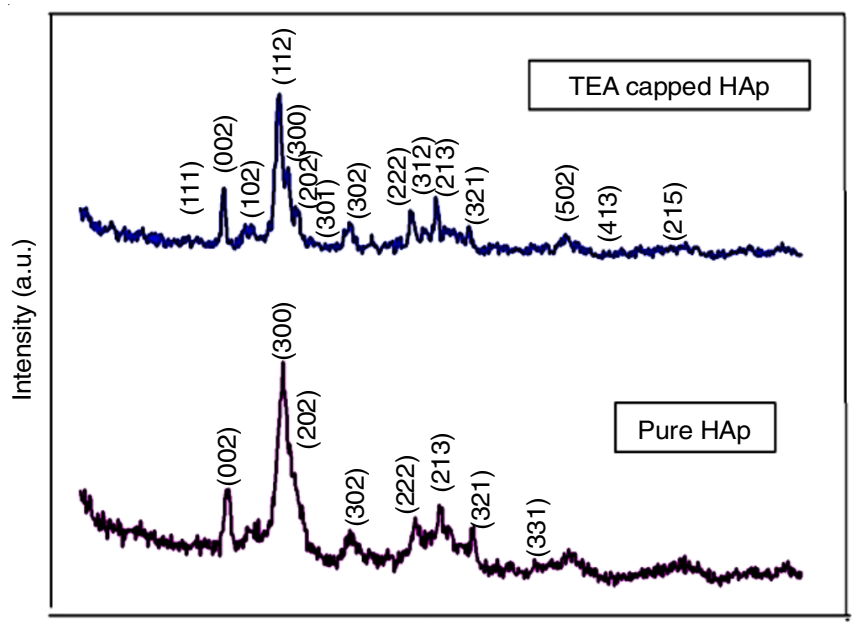

$2 \theta\left({ }^{\circ}\right)$

Fig. 2. XRD pattern of pure and triethanolamine capped hydroxyapatite nanoparticles

The crystallite size of pure hydroxyapatite is $42 \mathrm{~nm}$ and triethanolamine capped hydroxyapatite is $22 \mathrm{~nm}$. This result proves that the growth of hydroxyapatite is depressed by triethanolamine capping. The XRD pattern for pure hydroxyapatite and triethanolamine capped hydroxyapatite showed the characteristic peaks at $25.78^{\circ}, 32.90^{\circ}, 42.23^{\circ}, 46.72^{\circ}$ and $49.44^{\circ}$ shows the presence of hydroxyapatite nanoparticles in both the samples represents the hkl values of (002), (300), (302), (222), (213), respectively. The peaks and hkl values at $63.10^{\circ}(502), 66.42^{\circ}(413), 75.40^{\circ}(215)$ revealed the presence of $\mathrm{CH}$ molecule which is attributed to triethanolamine.

Scanning electron microscope analysis: SEM images predicts the direct information about the size and shape of the crystallinity in the as-synthesized samples. Fig . 3 shows the SEM and EDAX analysis of pure and triethanolamine capped hydroxyapatite nanoparticles. From the micrograph, pure hydroxyapatite reveals spherical shaped morphology with large aggregated particles was observed and triethanolamine capped hydroxyapatite shows the presence of nanorods. The average particle size ranges from 40 to $70 \mathrm{~nm}$ in pure hydroxyapatite and 50 to $70 \mathrm{~nm}$ for triethanolamine capped hydroxyapatite.

Energy dispersive X-ray analysis was performed to identify the elemental composition of pure hydroxyapatite and triethanolamine capped hydroxyapatite nanoparticles. EDAX pattern indicates the presence of $\mathrm{Ca}, \mathrm{P}$, and $\mathrm{O}$ in as-synthesized nanoparticles which indicate the purity of samples. Sharp calcium and phosphorous peaks are present in EDAX spectra. EDAX analysis revealed that $\mathrm{Ca} / \mathrm{P}$ molar ratios were 1.668 and 1.654 , respectively. The ratio perfectly matches with the standard value 1.6.
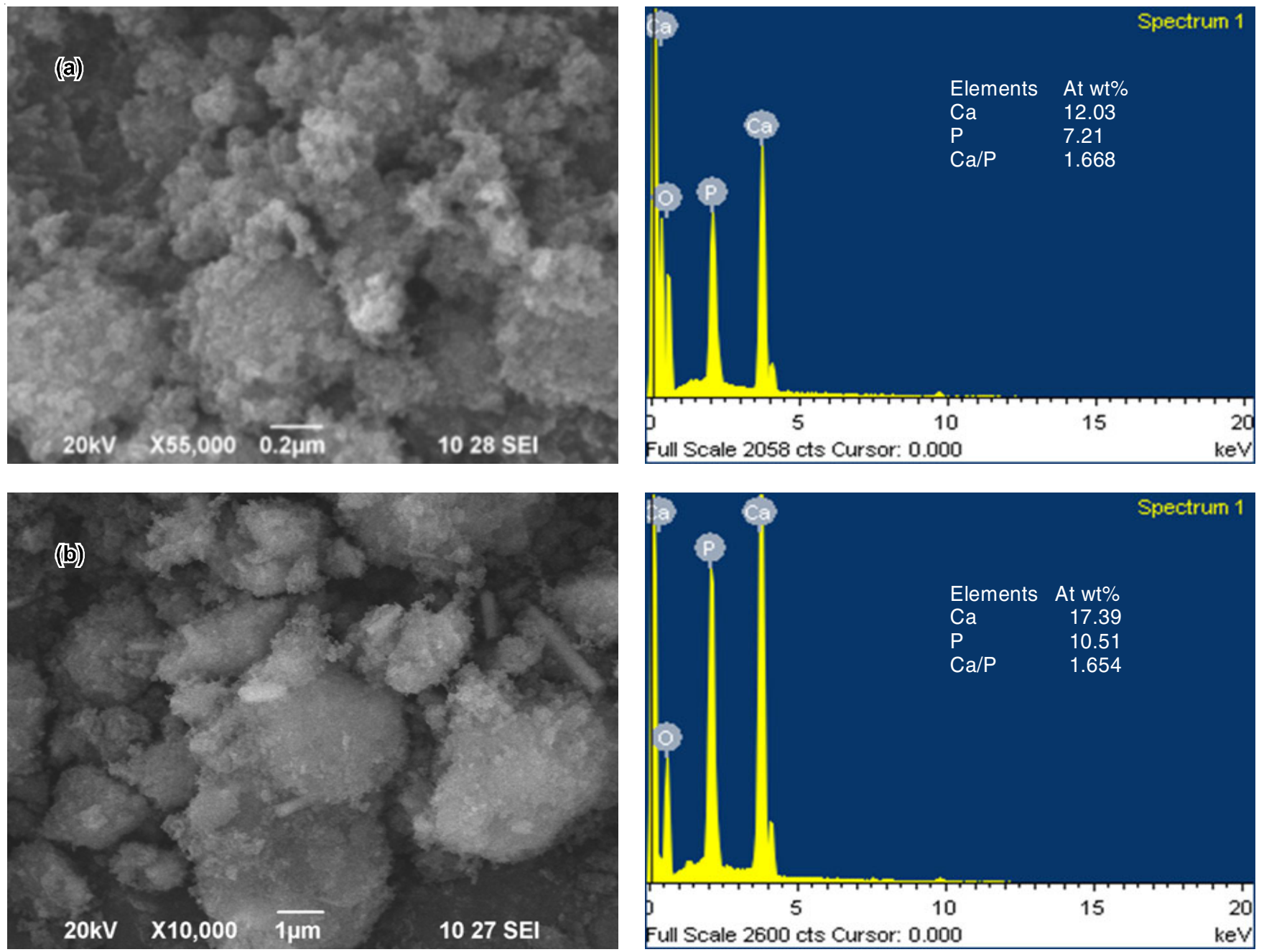

Fig. 3. SEM and EDAX analysis of (a) pure hydroxyapatite and (b) triethanolamine capped hydroxyapatite nanoparticles 

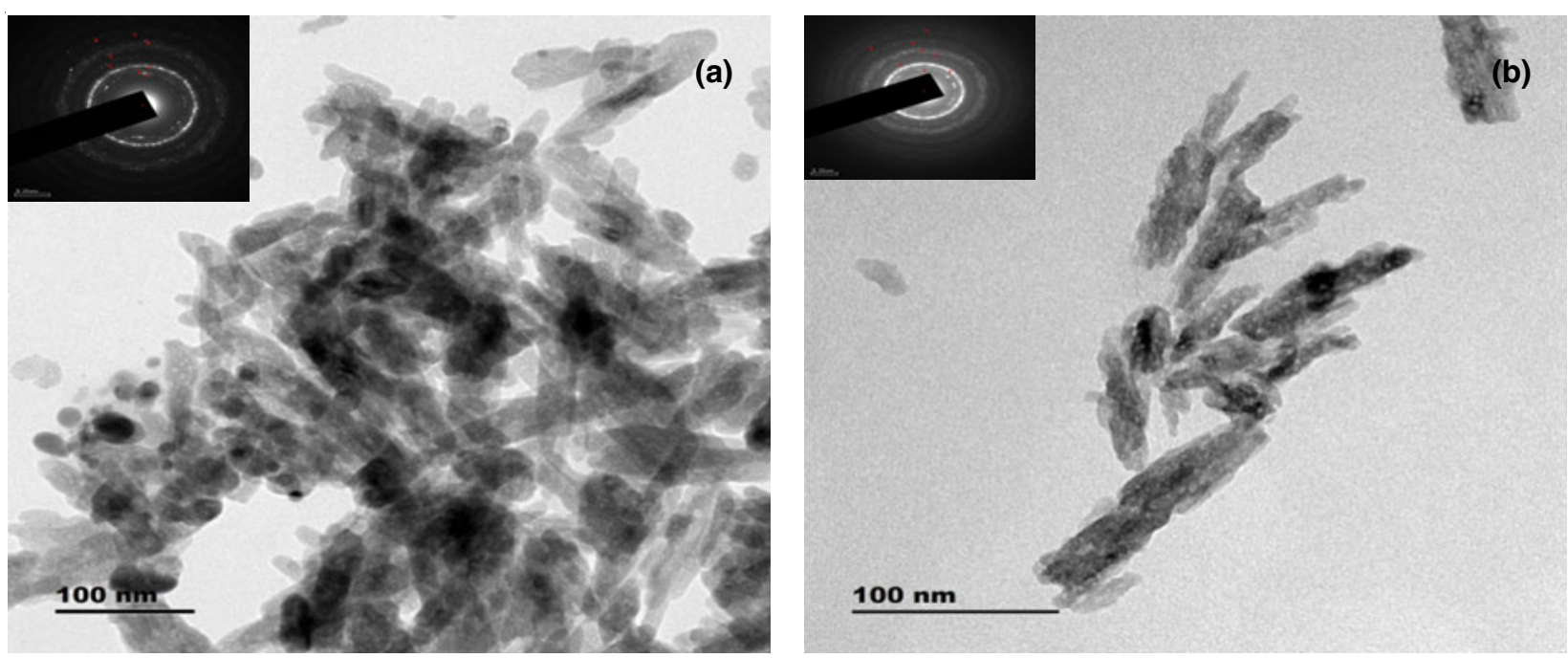

Fig. 4. TEM images and SAED pattern of (a) pure hydroxyapatite and (b) triethanolamine capped hydroxyapatite

Transmission electron microscopy: Fig. 4 depicted the composite morphology of the samples by TEM and selected area diffraction pattern (SAED) of pure hydroxyapatite and triethanolamine capped hydroxyapatite. The SAED pattern of all the samples exhibits continuous rings around the sharp spot, which shows the polycrystalline nature of prepared samples. When triethanolamine is capped with pure hydroxyapatite, it effectively controls the growth of hydroxyapatite nanoparticles. Both the samples show clear rod shaped morphology. Pure hydroxyapatite shows agglomerated structures whereas triethanolamine capped hydroxyapatite shows clear and separate rods.

Antibacterial activity: The potential effect of the synthesized samples towards the microbial pathogens was explored by agar well diffusion method. By using zone of inhibition method, antibacterial activities were calculated for $S$. aureus and $E$. coli bacterial strains with reference to standard drug ciproflaxin. Both samples had shown a considerable antibacterial activity against Gram positive and Gram negative bacterial strains. The zone of inhibition of pure hydroxyapatite nanoparticles against $S$. aureus was $15 \mathrm{~mm}$ and E. coli was found to be $18 \mathrm{~mm}$. Though the zone of inhibition was found to be less than the standard antibiotic ciproflaxin, the particles synthesized in the current study could be applied as biocompatible material and the obtained property itself may prevent the infection when the particles are applied as biomaterials. The hydroxyapatite nanoparticles may have a good capacity to disrupt the bacterial cell membrane and was occupied with positive zeta potential on its surface [12]. So the particles can actively participate in damaging the bacterial cell membrane where cytoplasmic content in bacterial cells had decreased and created the cell death. The zone of inhibition is given in Table- 1 and is the mean of diameter of zone of inhibition with three replicates.

Cytotoxity test: The cytotoxicity study for triethanolamine capped hydroxyapatite was worked out in human embryonic kidney cell line HEK 293. As in vitro studies are simple, miniaturized, avoids ethical issues and high throughput screening method and is the preliminary confirmation for effective application in in vivo, the cytotoxicity effect of the particles were carried out using cell line in the current study. The HEK 293 cell line was incorporated in capped hydroxyapatite nanopart-
TABLE-1

ZONE OF INHIBITION FOR Staphylococcus aureus, Escherichia coli OF PURE AND TRIETHANOLAMINE CAPPED HYDROXYAPATITE

\begin{tabular}{lccc}
\hline \multirow{2}{*}{ Test organisms } & \multicolumn{3}{c}{ Zone of inhibition in diameter $(\mathrm{mm})$} \\
\cline { 2 - 4 } & $\begin{array}{c}\text { Ciproflaxacin } \\
(10 \mu \mathrm{g} / \mathrm{disc})\end{array}$ & $\begin{array}{c}\text { Pure } \\
\text { HAp }\end{array}$ & $\begin{array}{c}\text { TEA capped } \\
\text { HAp }\end{array}$ \\
\hline Staphylococcus aureus & 40 & 15 & 12 \\
Escherichia coli & 30 & 18 & 12 \\
\hline
\end{tabular}

icles for 12,32 and 48 with the concentration of 12.5, 25, 50, 100 and $200 \mu \mathrm{g}$, respectively and maintained at $37^{\circ} \mathrm{C}, 50 \%$ carbon dioxide, air at $95 \%$ and relative humidity $100 \%$. After $24 \mathrm{~h}$, all the samples are treated with the cell line.

The cytotoxicity study against L929 mouse fibroblast revealed that cell viability is directly proportional to concentration but in kidney cell line HEK293, low concentration of the particles revealed the negligible toxicity and high concentration revealed low toxicity. The cytotoxicity study against HEK 293 cell line revealed that cell viability is directly proportional to concentration. The concentration $v s$. cell growth data is given in Table2. Similarly, incubation time and concentration increases will cause decrease in cell growth (Table-3).

TABLE-2

CONCENTRATION $v s$. CELL GROWTH OF TRIETHANOLAMINE CAPPED HYDROXYAPATITE

\begin{tabular}{cc}
\hline Concentration $(\mu \mathrm{g} / \mathrm{mL})$ & Cell growth $(\%)$ \\
\hline 12.5 & 97.8 \\
25 & 93.8 \\
50 & 89.6 \\
100 & 81.4 \\
200 & 73.9 \\
\hline
\end{tabular}

From previous report, it was studied that when healthy cells are incorporated with nanoparticles; it affects the plasma membrane and causes lysis among the cells. After removing the nanoparticles from cell line the cell line is treated with MTT. MTT enters the mitochondria and reduces the cell line to an insoluble formazon product. Then stabilized with DMSO and absorbance are studied via microplate reader. The main factors in cell viability are grain size and chemical stability. From Fig. 

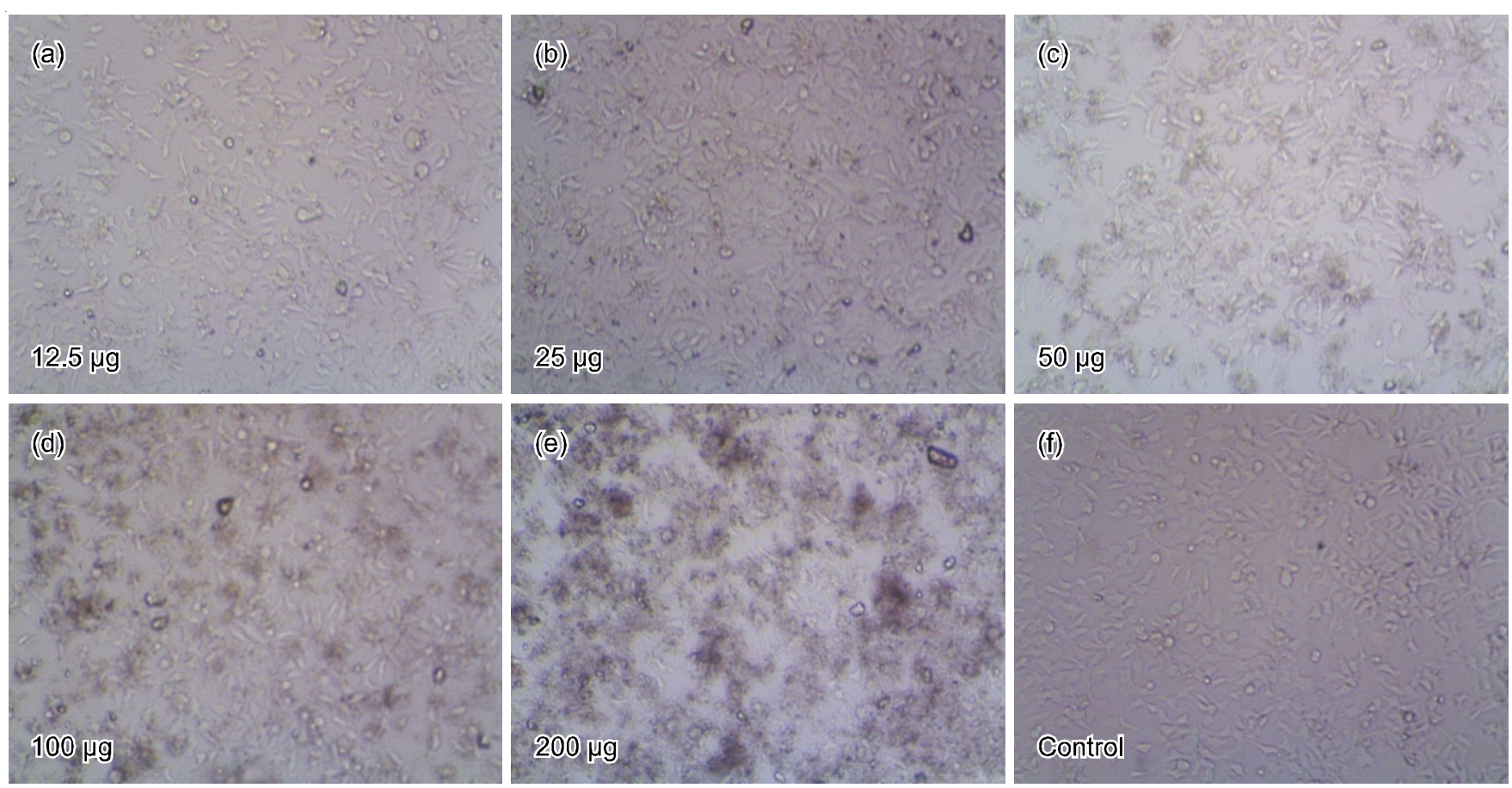

Fig. 5. Light microscopic images of HEK293 cell lines of triethanolamine capped hydroxyapatite nanaoparticles

\begin{tabular}{cc} 
TABLE-3 \\
CONCENTRATION $v s$. TIME TAKEN FOR CELL GROWTH OF \\
TRIETHANOLAMINE CAPPED HYDROXYAPATITE \\
\hline Concentration $(\mu \mathrm{g} / \mathrm{mL})$ & Toxicity $(\%)$ \\
\hline 12.5 & 0.3116 \\
25 & 0.2990 \\
50 & 0.2856 \\
100 & 0.2593 \\
200 & 0.2356 \\
\hline
\end{tabular}

$5(\mathrm{a}-\mathrm{f})$, it is almost clear that the nanoparticles have less mass concentration and total surface area compared to bulk materials. Bulk materials interact with wide area to exhibit stronger stimulus on cell surface to reduce cell viability, whereas it is directly proportional in nanoparticles. Due to non-toxic nature of hydroxyapatite nanoparticles there exhibits no inhibitory concentration value. Thus cytotoxicity of triethanolamine capped hydroxyapatite is biocompatible upto the concentration of 200 $\mu \mathrm{g}$. It also enumerates that biological effects are based on size, aggregation state, chemical composition and surface morphology of nanoparticles [15].

\section{Conclusion}

The pure and triethanolamine capped hydroxyapatite are prepared by microwave assisted synthesis. Nanocrystalline with hexagonal type structure was found in the XRD analysis. Triethanolamine capping does not influence the crystal structure but the average grain size was reduced. SEM results revealed the morphology of hydroxyapatite nanoparticles which significantly changed from spherical to rod shaped by triethanolamine capping. The EDAX results confirmed the presence of $\mathrm{Ca}, \mathrm{P}, \mathrm{O}$ in hydroxyapatite nanoparticles. The obtained hydroxyapatite nanoparticles exhibit antibacterial activity against E. coli and S. aureus making it to apply in clinical applications. Cytotoxicity results of HEK293 cell line predict the non-toxic efficacy of triethanolamine capped hydroxyapatite nanoparticles.

\section{REFERENCES}

1. D. Gopi, J. Indira, S. Nithiya, L. Kavitha, U.K. Mudali and K. Kanimozhi, Bull. Mater. Sci., 36, 799 (2013); https://doi.org/10.1007/s12034-013-0540-6.

2. H.M. Pandya and P. Anitha, J. Environ. Nanotechnol., 3, 101 (2013); https://doi.org/10.13074/jent.2013.12.132058.

3. N. Rameshbabu, T.S. Sampath Kumar, T.G. Prabhakar, V.S. Sastry, K.V.G.K. Murty and K.P. Rao, J. Biomed. Mater. Res., 80A, 581 (2006); https://doi.org/10.1002/jbm.a.30958.

4. N. Iqbal, M.R.A. Kadir, N.H. Mahmood, N. Salim, G.R.A. Froemming, H.R. Balaji and T. Kamarul, Ceram. Int., 40, 4507 (2014); https://doi.org/10.1016/j.ceramint.2013.08.125.

5. Y. Li, C.T. Nam and C.P. Ooi, J. Phys. Conf. Ser., 187, 012024 (2009); https://doi.org/10.1088/1742-6596/187/1/012024.

6. V. Kalaiselvi, R. Mathammal and P. Anitha, Int. J. Adv. Sci. Eng., 4, 571 (2017); https://doi.org/10.29294/IJASE.4.2.2017.571-574.

7. N. Puvvada, P.K. Panigrahi, H. Kalita, K.R. Chakraborty and A. Pathak, Appl. Nanosci., 3, 203 (2013); https://doi.org/10.1007/s13204-012-0133-5.

8. P. Anitha and H.M. Pandya, Nanotechnol. Res. Prac., 3, 120 (2014); https://doi.org/10.13187/ejnr.2014.3.120.

9. P. Kanchana and C. Sekar, Mater. Sci. Eng. C, 42, 601 (2014); https://doi.org/10.1016/j.msec.2014.05.072.

10. V. Kalaiselvi and R. Mathammal, Int. J. Mag. Eng. Technol. Manage. Res., 3, 133 (2015).

11. G.S. Kumar, E.K. Girija, M. Venkatesh, G. Karunakaran, E. Kolesnikov and D. Kuznetsov, Ceram. Int., 43, 3457 (2017); https://doi.org/10.1016/j.ceramint.2016.11.163.

12. M. Manoj, R. Subbiah, D. Mangalraj, N. Ponpandian, C. Viswanathan and K. Park, Nanobiomedicine, 2, 2 (2015); https://doi.org/10.5772/60116.

13 D. Shankar, S. Basker and S. Karthik, Asian J. Pharm. Clin. Res., 10, 46 (2017); https://doi.org/10.22159/Ajpcr.2017.V10i11.20226.

14 A.R. Shahverdi, A. Fakhimi, H.R. Shahverdi and S. Minaian, Nanomed.: Nanotechnol., Biol. Med., 3, 168 (2007); https://doi.org/10.1016/j.nano.2007.02.001

15 F. Martinez-Gutierrez, P.L. Olive, A. Banuelos, E. Orrantia, N. Nino, E.M. Sanchez, F. Ruiz, H. Bach amd Y. Av-Gay, Nanomed.: Nanotechnol., Biol. Med., 6, 681 (2010); https://doi.org/10.1016/j.nano.2010.02.001. 